\title{
PCA-based Palm Vein Authentication System
}

\author{
Sahar Bayoumi \\ Information technology dept., \\ King Saud University \\ Saudi Arabia \\ $\underline{\text { sahali@ksu.edu.sa }}$ \\ Afnan Sheikh \\ Information technology dept, \\ King Saud University \\ Saudi Arabia \\ Afnan k.s@hotmail.com \\ Sulaf Al-Magooshi \\ Information technology dept., \\ King Saud University \\ Saudi Arabia \\ thesulaf@yahoo.com
}

\author{
Sara Al-Zahrani \\ Information technology dept., \\ King Saud University \\ Saudi Arabia \\ Sara alzhrani@hotmail.com \\ Ghada Al-Sebayel \\ Information technology dept, \\ King Saud University \\ Saudi Arabia \\ Ghada.sebayel@gmail.com \\ Sara Al-Sayigh \\ Information technology dept., \\ King Saud University \\ Saudi Arabia \\ s0so99@hotmail.com
}

\begin{abstract}
In this paper we proposed a system for authentication using Palm Vein based on using principle component analysis (PCA) for feature extraction. Palm vein images of dorsa captured using infrared camera. PCA is applied to generate vector of features that represent the highest detailed variant information. A matching process is then applied to find the best match from the data base to recognize and authenticate the person. Experiments show that our system able to recognize human with accuracy $85 \%$ in real-time based on supervised recognition.
\end{abstract}

Keywords-Biometrics ; Securtiy ; Image processing ; Palm vein Authentication; principle component analysis (PCA); supervised pattern recognition.

\section{INTRODUCTION}

With the increasing importance of information security in every aspect of our lives, many ideas were introduced for identification and authentication such as: password, ID cards, behavioral, biometrics. Biometric technology is becoming the base approach to fulfill the need of accurate recognition systems and access control methods. It is based on recognizing people based on their physiological characteristics such are fingerprint, face, hand/finger geometry, iris, retina, signature, gait, voice, hand vein, odor or the DNA information. It is indispensable s element in several areas such as airports, banking, hospital security and access to restricted areas.

A palm vein characteristic has gained more interest in authentication because of its uniqueness even between twins. Furthermore, it will not vary during the person's lifetime and impossible to read or copy since it lies under the skin. The most important advantage of palm vein is that it is exist only for live humans.

In this paper we proposed a system for authentication using hand dorsa palm vein as biometric features based on PCA.

We applied PCA on each image of the training set instead of building a matrix of all the training set. Our choice aims to prove variance between features within the same image will provide good Eigenveins that support good recognition.

Following sections are organized to show related work in section II followed by description of the implemented approaches in section III, Results are then presented in section IV and finally conclusion and future work in section V.

\section{RELATED WORK}

Wang and Leedham used thermal-imaged vein pattern of the hand dorsa in developing verification system based on following stages : Hand Image Acquisition, Image enhancement, Vein Pattern Segmentation, Skeletonization and Matching. They used shapes of the preprocessed vein patterns by calculating their line segment Hausdorff distances [1].

Wang and Chen proposed algorithm for palm vein capturing system that can effectively delimit the hand region from the background to extract the vein pattern [2]

Zhang et al proposed a scheme of personal authentication using low cost CCD camera for capturing infrared palm images of the bach of the hand. They build system based extracting ROI from the captured image [3] 
Shahin et al, presented a hand vein authentication system using fast spatial correlation of hand vein patterns. The designed system was tested for verification purpose only over a dataset collected with the designed prototype system. Proposed system conclude that the hand vein patterns are unique for each identity and even between right and left hands for same person[4].

Mirmohamadsadeghi and Drygajlo proposed recognition of palm vein based on local texture patterns. They used Local Binary Patterns and Local Derivative Patterns as features based on their ability to adapt to palm vein description [5].

\section{METHODLIGY}

\section{A. Data Collection}

M2-PalmVein ${ }^{\mathrm{TM}}$ palm vein scanner from M2SYS which uses a near-infrared light to capture the hand dorsa vein map to be used later in the authentication process, as shown in Figure 1.

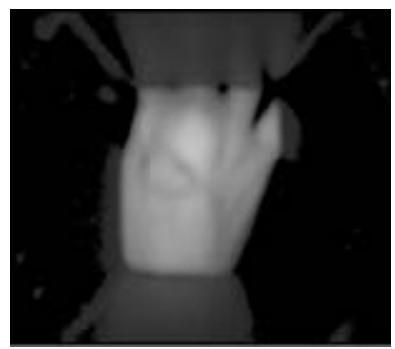

Figure 1: Image of hand dorsa palm vein with M2- palm vein scanner.

\section{B. Feature Extraction Using PCA}

PCA is a mathematical procedure that uses an orthogonal transformation to convert a set of possibly correlated $\mathrm{M}$ variables into a set of uncorrelated variables called principal components $\mathrm{K}$, in which $\mathrm{K}$ is always less than or equal $\mathrm{M}$ [ Its major use is as a tool in exploratory data analysis as well as making predictive models. Its operation can be thought of as revealing the internal structure of the elements that detains the major features which best explains the variance in the data [6].

We applied PCA on each image of the training set instead of building a matrix of all the training set. Our choice aim to prove variance between features within the same image will provide good Eigenveins that support good recognition.

Following steps are applied on captured images in order to extract Eigenveins:

$\$$ Normalization will be performed to change the range of pixel intensity values.

\& The covariance matrix will be calculated from the normalized image to identify the most discriminate features in the image.

* The eigenvalues and eigenvectors are calculated using the covariance matrix.

* For each MxM image, K Eigenveins are selected as discriminant features based on the eigenvalues weights.
Figure 3 show weights of eigenvectors for a sample of three images captured for different persons. From experimental results and as shown in Figure 2, the first three eigenvalues holding about $95 \%$ of the significant information. Therefore, the associate three eigen vectors are selected to represent the Eigenveins.

Since images are dimensioned 480x640, the Eigenveins will be a vector of 1920 features to be used in identification process,

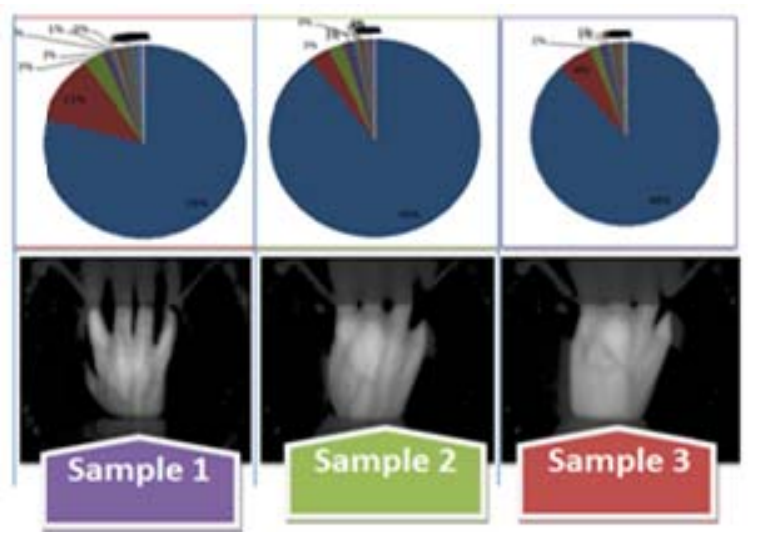

Figure 2: sample images used to determine the value of $K$

\section{Identification:}

A training set of palm vein images are collected and its eignveins are stored in a data base to be used for identification. Identifying new captured palm vein will be based on one-to-many matching process where simple matching process is applied. A Euclidean distance between to between unknown pattern and known patterns that stored in the data based are calculated. Then unknown pattern are identified to the one with the minimum distance.

\section{RESULTS}

A total of 100 palm vein images are collected from 20 different persons aged between 25- 40 years old, each recorded 5 images. They all used for training the system and data based build.

Testing are run using real-time images where 20 persons have been involved considering that two of them are not part of the training set images. During testing, each unknown person will be introduced to the system twice either he/she identified or not identified in the first attempt.

System results showed that $85 \%$ of the unknown patterns are positively identified and authorized by the system where $76 \%$ are identified from the first attempt while $24 \%$ from the second attempt. 
Those who are identified from the second attempt are not adjusting their hands correctly over the infrared camera. Also, change in illumination can cause negative identification

\section{CONCLUSION AND FUTURE WORK}

We proposed a system for authentication using palm vein as a biomedical features based on Principal Component Analysis (PCA). In our system, we used PCA to discriminate the variances between the image features instead of between the whole training set.

Our system was able to identify $85 \%$ of the unknown patterns in real-time which considered good results regarding to the local PCA features.

As a future work, we are planning to improve the system accuracy by generating more features and enlarge the training set. We also, plan to extend our application by adding a smart card device that store the extracted palm vein feature of each user. This will change the identification and validation process manner to one to one matching therefore improve the system's response time.

One useful way of using this system is to implement an automated attendance management system in the university, where students are registered into the system by instructors. They system would help each instructor managing the attendance of students in his ther class as well as providing the desired statistics and access information.

\section{AKNOWLEGMENT}

The authors extend their appreciation to the Deanship of Scientific Research at King Saud University for funding the work through the research group project RGP-VPP-157".

\section{REFERENCES}

[1] Lingyu Wang and Graham Leedham ." A Thermal Hand Vein Pattern Verification System"S.Singh et al.(Eds.):ICAPR 2005 , LNCS 3687, pp. 58-65, 2005.

[2] Jing-Wein Wang and Tzu-Hsiung Chen. "Building Palm Vein Capturing system for Extraction". $21^{\text {st }}$ International conference on system engineering, 2011.

[3] Yi-Bo Zhang, Qin Li, Jane You and Prabir Bhattacharya. "Palm Vein Extraction and Matching for personal authentication" VISUAL 2007, LNCS 4781, pp. 154-164, 2007.

[4] Mohamed Shahin, Ahmed Badawi, and Mohamed Kamel . " Biometric Authentication Using Fast Correlation of Near Infrared Hand Vein Patterns" International Journal of Biological and Life Sciences 2:3 2006 .

[5] Leila Mirmohamadsadeghi and Andrzej Drygajlo. "Palm Vein Recognition with local binary patterns and local derivative patterns" International joint conference on biometrics, 2011.

[6] M.Heenaye-Mamode Khan, R.K. Subramanian, and N. A. Mamode Khan . "Low Dimensional Representation of Dorsal Hand Vein Features Using Principle Component Analysis (PCA)". World Academy of Science, Engineering and Technology 252009

[7] "Palm vein biometric systems", biometric news portal. [Online].Available:http://www.biometricnewsportal.com/palm _biometrics.asp 